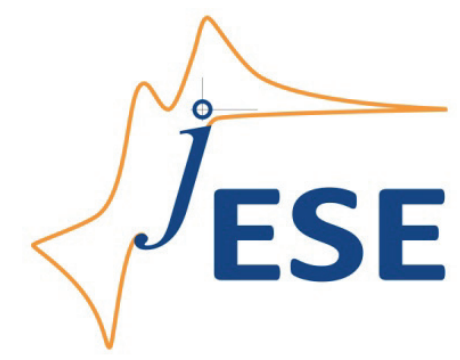

Open Access : : ISSN 1847-9286

www.jESE-online.org

Original scientific paper

\title{
Formation of the honeycomb-like electrodes by the regime of pulsating overpotential in the second range
}

\author{
NEBOJŠA D. NIKOLIĆ $₫$, GORAN BRANKOVIĆ* and MIOMIR G. PAVLOVIĆ
}

ICTM-Institute of Electrochemistry, University of Belgrade, Njegoševa 12, 11000 Belgrade, Serbia

*Institute for Multidisciplinary Research, University of Belgrade, Kneza Višeslava 1a, Belgrade, Serbia

${ }^{凶}$ Corresponding Author: E-mail: nnikolic@tmf.bg.ac.rs; Tel./Fax: +381 113370389

Received: January 27, 2012; Revised: February 21, 2012; Published: March 22, 2012

\begin{abstract}
In this study the honeycomb-like copper structures electrodeposited by the regime of pulsating overpotential in the second range were analyzed by the technique of scanning electron microscopy. The overpotential amplitude of $1000 \mathrm{mV}$, deposition pulse of $1 \mathrm{~s}$, and pause durations of 1, 5, 10 and $15 \mathrm{~s}$ were selected for the production of this type of structures. The size of holes which remained upon detachment of hydrogen bubbles do not depend on the length of pause duration. On the other hand, the change in morphology of electrodeposited copper around holes from cauliflower-like agglomerates of copper grains to degenerated dendrites is observed when pause duration was increased. Effects of the application of the regime of pulsating overpotential in the second range on the formation of the honeycomb-like structures were less pronounced than the effects attained by the application of the same regime in the millisecond range. However, they were more pronounced than those attained by electrodeposition in the regime of constant potential.
\end{abstract}

\section{Keywords}

Copper; electrodeposition; pulsating overpotential; hydrogen; scanning electron microscope.

\section{Introduction}

Open porous structures with an extremely large surface area are of high technological significance, because they are very suitable as electrodes in many electrochemical devices, such as fuel cells, batteries and sensors [1,2], and in catalysis [3]. Electrodeposition technique was shown to be very favorable way for the production of this type of electrodes $[1,2,4]$. Open porous copper 
electrodes, denoted as both 3-D foam [1,2,4] or honeycomb-like electrodes [5-13], are formed by electrodeposition at high current densities and overpotentials, when, parallel to electrodeposition process, the hydrogen evolution reaction occurs. The main characteristics of these electrodes are holes or pores formed upon detachment of hydrogen bubbles, surrounded by the agglomerates of metal grains or dendritic particles. This way of the preparation of porous electrodes is denoted as gas bubble dynamic template method, since the hydrogen bubbles function as a dynamic template for the formation of this type of deposits. The specific surface area of these structures is determined by the number and size of the holes, as well as by the width of the walls in-between [4]. Aside from copper, which is the most studied system [1,2,4-15], open porous structures of some other technologically important metals, such as tin [1], nickel [16], silver [17,18], gold [19], lead [20], and copper-tin alloys [2], were also investigated.

The conditions of the formation of open porous copper electrodes by constant regimes of electrolysis (the potentiostatic and galvanostatic ones) are well examined and systematized $[1,13]$. Two ways have been proposed for the increase in specific surface area and the improvement of micro- and nano-structural characteristics of the open porous structures: a) the addition of additives to the electroplating baths [4,21-23] and b) the application of periodically changing regimes of electrolysis, such as those of pulsating overpotential (PO) [24-27], pulsating current (PC) [28-30] and reversing current (RC) [31].

All benefits in the formation of the honeycomb-like structures by the electrochemical deposition processes at periodically changing rate were reported for the cases of electrodepositions in the millisecond range. However, the application of the periodically changing regimes of electrolysis on the formation of open porous structures in the second range of pulsating is completely unknown. Therefore, the aim of this paper is to analyze the formation of the honeycomb-like structures by the regime of pulsating overpotential (PO) in the second range.

\section{Experimental}

Copper was electrodeposited from $0.15 \mathrm{M} \mathrm{CuSO}_{4}$ in $0.50 \mathrm{M} \mathrm{H}_{2} \mathrm{SO}_{4}$, in an open glass cylindrical cell of $100 \mathrm{~mL}$ at the room temperature. Doubly distilled water and analytical grade chemicals were used for the preparation of the solution for electrodeposition of copper.

Copper electrodeposition was performed by the square-wave pulsating overpotential (PO) technique. In all experiments, the overpotential amplitude of $1000 \mathrm{mV}$ was used. Pause durations of $1,5,10$ and $15 \mathrm{~s}$ were applied, while the applied deposition pulse was $1 \mathrm{~s}$.

The working electrodes used for electrodeposition of copper were cylindrical copper wires. The diameter of these copper electrodes was $0.08 \mathrm{~cm}$, while their length was $2 \mathrm{~cm}$. The counter electrode was copper foil of $0.80 \mathrm{dm}^{2}$ surface area placed tightly to the walls of the cell, whereas the reference electrode was copper wire, the tip of which was positioned at the distance of $0.2 \mathrm{~cm}$ from the surface of the working electrode. The working electrode was always situated in the centre of cell.

The preparation of working electrodes consisted of alkaline degreasing at $70^{\circ} \mathrm{C}$ followed by an acid etching $\left(20 \% \mathrm{H}_{2} \mathrm{SO}_{4}\right)$ at $50^{\circ} \mathrm{C}$. After each of these preparation phases, working electrodes were rinsed by distilled water. This pretreatment of the electrodes was important in order to obtain the same state of initial electrode surface prior to each experiment.

Copper was electrodeposited by consuming $10 \mathrm{mAh} \mathrm{cm}^{-2}$ of electricity.

The obtained copper deposits were examined by a scanning electron microscope TESCAN Digital Microscopy. 


\section{Results and Discussion}

Figure 1 shows the honeycomb-like structures obtained by the regime of the pulsating overpotential (PO) in the second range. For the formation of the shown honeycomb-like structures, the following parameters of the PO regime were applied: the overpotential amplitude of $1000 \mathrm{mV}$, the deposition pulse of $1 \mathrm{~s}$, and the pause durations of 1 (Fig. 1a), 5 (Fig. 1b), 10 (Fig. 1c) and $15 \mathrm{~s}$ (Fig. 1d). It can be noticed from Fig. 1 that there is no significant difference in the number of holes formed upon detachment of hydrogen bubbles from the electrode surface. The number of the formed holes was estimated to be $125 \pm 5$ holes per $\mathrm{mm}^{2}$ surface area of the copper electrode.

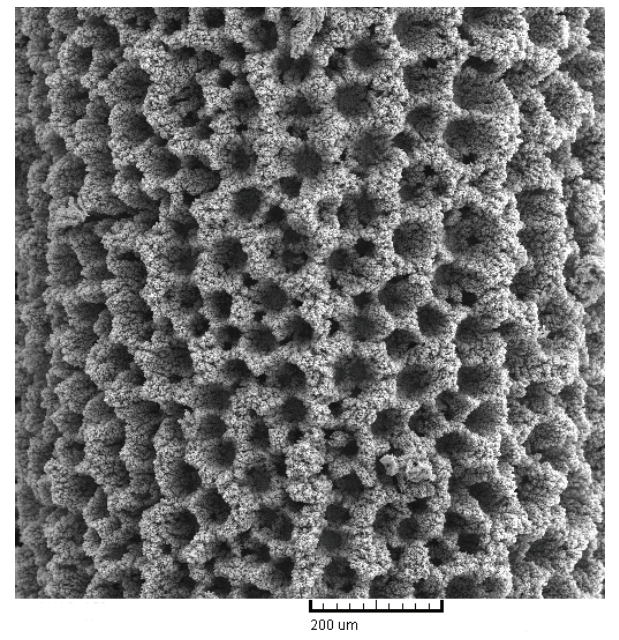

a)

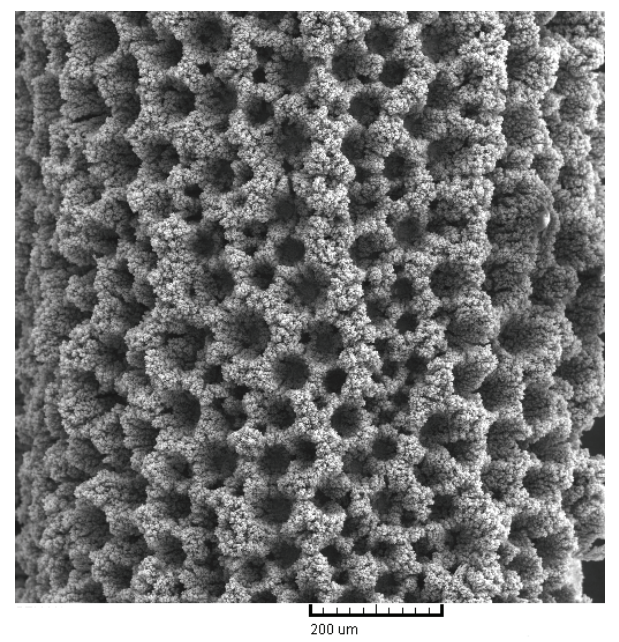

c)

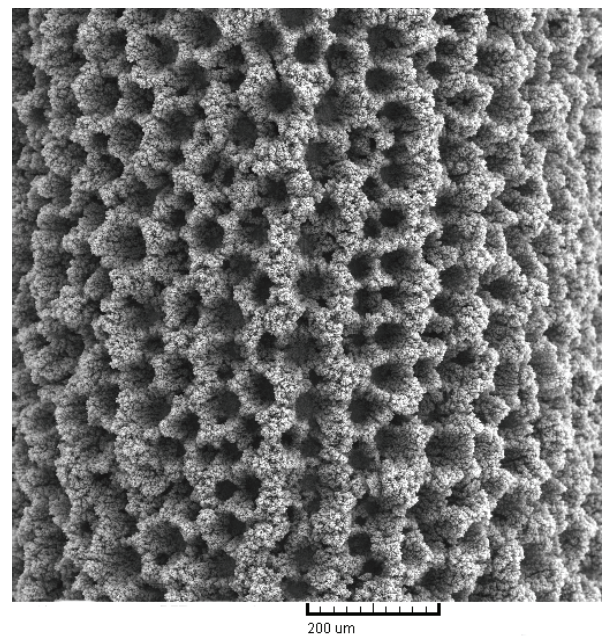

b)

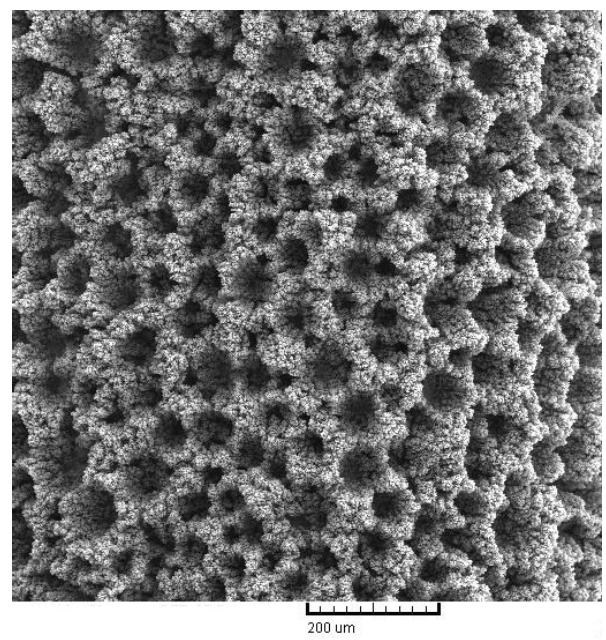

d)

Figure 1. The honeycomb-like copper structures obtained by the regime of pulsating overpotential $(P O)$ in the second range with pause durations of: a) 1, b) 5, c) 10 and d) $15 \mathrm{~s}$.

The overpotential amplitude: $1000 \mathrm{mV}$. Deposition pulse: $1 \mathrm{~s}$.

The detailed analysis of these honeycomb-like electrodes showed negligible presence of holes obtained by the coalescence of closely formed hydrogen bubbles. The analysis of the holes at higher magnifications showed that their size does not depend on the pause duration. The typical hole, formed by detachment of a hydrogen bubble, which was obtained with pause duration of $5 \mathrm{~s}$ is shown in Fig. 2. The average hole size was estimated to be $80 \pm 2.0 \mu \mathrm{m}$. 


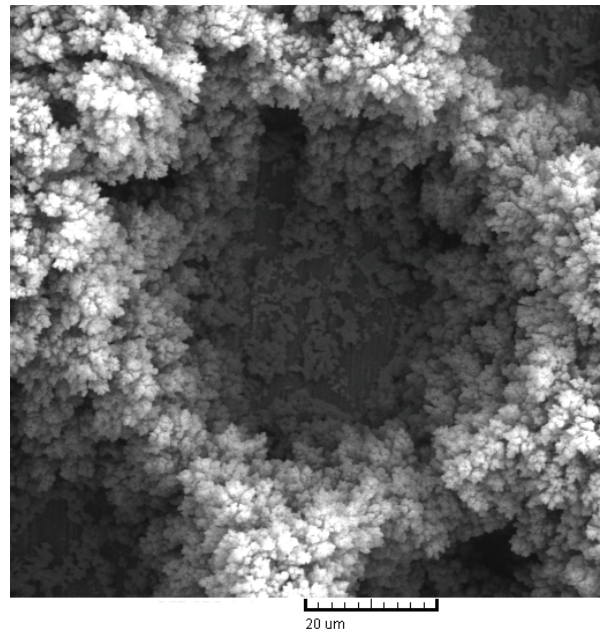

Figure 2. The typical hole obtained by the PO regime with pause duration of $5 \mathrm{~s}$. The overpotential amplitude: $1000 \mathrm{mV}$. Deposition pulse: $1 \mathrm{~s}$.

Morphologies of copper deposits formed around holes are shown in Fig. 3. It can be seen in Fig. 3 that macro-structure of the obtained deposits strongly depends on the pause duration. The cauliflower-like agglomerates of copper grains were formed with pause durations of 1 (Fig. 3a) and $5 \mathrm{~s}$. The increase in pause duration to 10 and $15 \mathrm{~s}$ led to the formation of deposits with dendritic characteristics (Figs. $3 b-3 d$ ). Dendritic character is more pronounced for the copper deposit obtained with pause duration of $15 \mathrm{~s}$ (Figs. 3c and 3d) than with the one that lasted $10 \mathrm{~s}$ (Fig. 3b). These morphological forms can be denoted as degenerate dendrites. Contrary to the strong effect of the pause duration on the macro-structure of the honeycomb-like electrodes, their microstructure was only negligibly affected. Both degenerate dendrites and cauliflower-like agglomerates of copper grains consist of small copper grains agglomerates. The size of grains of both, the cauliflower-like agglomerates of grains and the degenerate dendrites, approached the nano-size dimensions.

Generally, the two types of pores (or holes) were formed by hydrogen evolution reaction during electrodeposition process [32]. The first type relates to macro-pores (or holes) formed upon detachment of hydrogen bubbles. These pores originate from the hydrogen bubbles formed in the initial stage of electrodeposition process. They are responsible for the creation of the honeycomblike structure and they, upon detachement from the electrode surface, contribute neither to the stirring of the solution nor to the change in hydrodynamic conditions in the near-electrode layer.

The origin of the second type of pores is related to the hydrogen bubbles generated at the peaks of agglomerates of copper grains during the growth process (the current density distribution effect). The agglomerates of copper grains were formed at the electrode surface in the initial stage of electrodeposition with hydrogen bubbles. Some of these freshly formed hydrogen bubbles will coalesce with hydrogen bubbles formed in the initial stage of electrodeposition leading to the growth of the former as the electrolysis proceeds (the increase in the size of macro-pores). Simultaneously, some of newly formed hydrogen bubbles will not coalesce with primarily formed hydrogen bubbles because they are situated between freshly formed copper nuclei, which represent a barrier for the coalescence with the hydrogen bubbles formed in the initial stage of electrodeposition, or for development of larger hydrogen bubbles. These newly formed hydrogen bubbles will detach from the electrode surface very fast, thus forming micro- and nano-pores through the interior of the deposit. "The stream of hydrogen" is formed of these detached small 
hydrogen bubbles, which causes the stirring of the solution and the change in hydrodynamic conditions in the near-electrode layer. The channel structure through the interior of the deposit was formed of this micro- and nano-pores.

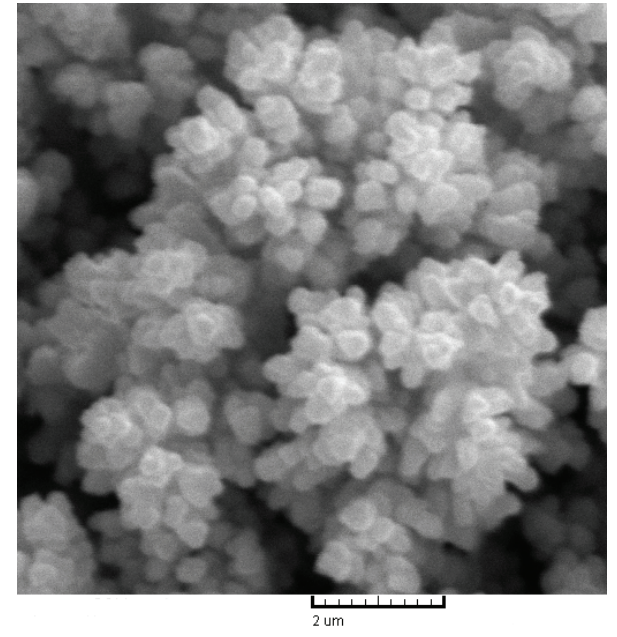

a)

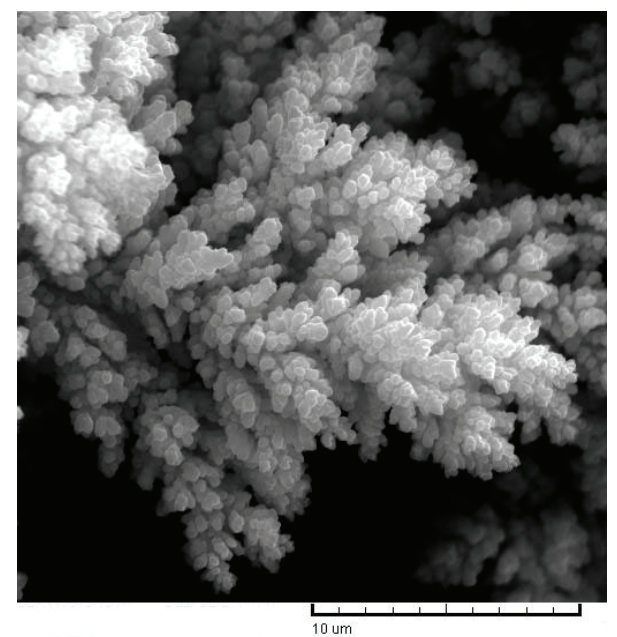

c)

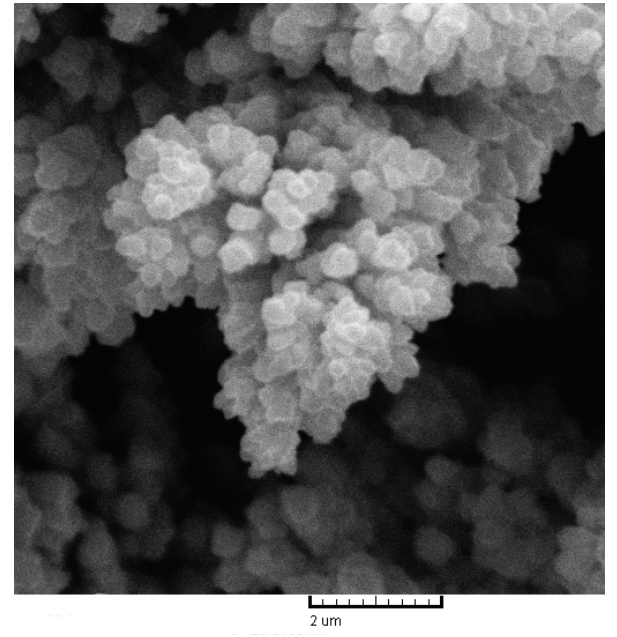

b)

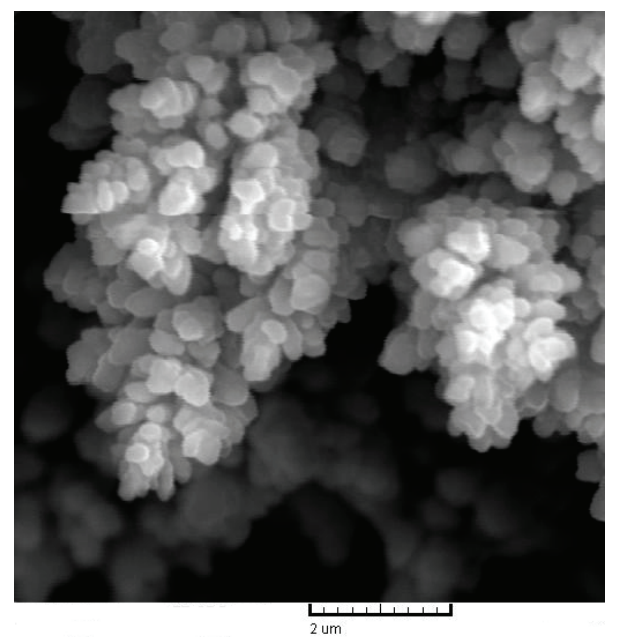

d)

Figure 3. Morphology of electrodeposited copper formed around holes by the PO regime with pause durations of: a) 1, b) 5, c) 10 and d) $15 \mathrm{~s}$.

The following conveniences in the formation of the honeycomb-like electrodes were achieved by the application of the PO regimes in the millisecond range in relation to the constant potentiostatic regime [24-27]:

a) the improvement of the current density distribution. Due to both, suppression of coalescence of closely formed hydrogen bubbles and the prevention of hydrogen bubbles to remain captive in the interior of deposit, the increase in the number of holes formed by detachment of hydrogen bubbles was observed;

b) the decrease in the amount of evolved hydrogen contributing to the effective stirring of the solution in the near-electrode layer. The remarkable change in surface morphology around holes from cauliflower-like agglomerates of metal grains to branchy dendrites was observed due to the decrease of this amount of evolved hydrogen.

Both of these conveniences result from the dissolution process caused by the anodic current density during the pause. It is clear that the condition (a) is fulfilled by the application of the PO 
regime in the second range (the increase of number of holes formed from detached hydrogen bubbles), while condition (b) is partially fulfilled (the mild change of surface morphology around holes). The possible reason for the smaller influence of the regime in the second range on the morphology of the honeycomb-like structures lies in the fact that the current during the pause is negligibly small in comparison with the current during deposition pulse. On the other hand, deposition pulses in the second range are sufficiently long for the current response during these pulses to approach the response of the constant potentiostatic regime. This increased difference between cathodic and anodic currents prevents the negligibly small anodic current during the pause to make significant effect on the dissolution process and hence on the amount of evolved hydrogen and surface morphology around the holes.

It is very clear from the previous consideration that the specific surface area of the honeycomblike electrodes obtained in the second range is larger than the one obtained in the constant potential mode. The number of the holes obtained by the potentiostatic electrodeposition at an overpotential of $1000 \mathrm{mV}$ (on the same type of working electrodes) was 45 per $\mathrm{mm}^{2}$ of electrode surface, while the average hole size was about $115 \mu \mathrm{m}$ [24].

It is necessary to note that during the formation of the honeycomb-like structures by the PO regime in both millisecond and second range with pause durations longer than deposition pulse, the parameters of the PO regime affect neither the number nor the size of the holes formed by detachment of hydrogen bubbles.

The concept of "effective overpotential" can be proposed to explain the formation of honeycomb-like structures by the PO regime in the range of seconds. This concept was originally proposed to explain the change in surface morphology during copper electrodeposition in the constant potentiostatic regime at high overpotentials, when, parallel to copper electrodeposition, the vigorous hydrogen evolution occurs $[5,13]$. According to this concept, when hydrogen evolution is vigorous enough, then electrodeposition process occurs at an overpotential which is effectively lower than the specified one, and this overpotential is denoted as "effective" in a deposition process. From the morphological point of view, this means that morphologies of metal deposits become similar to those obtained at some lower overpotentials at which hydrogen evolution does not occur or it is very slow. This concept can be adapted for the case of electrodeposition by the PO regime and denoted as "effective overpotential amplitude" concept. The appearance of degenerate dendrites clearly indicates the increase in the degree of diffusion control with the increase in pause duration.

Anyhow, morphological characteristics of the honeycomb-like structures obtained by the PO regime in the second range were between those obtained by the constant potential regime and by the PO regime in the millisecond range. Although the improvement of micro- and nano-structural characteristics of the honeycomb-like electrodes achieved by the application of the PO regime in the second range was smaller than the one achieved by the application of the same regime in the millisecond range, it is clear that the useful effects of the application of this electrolysis regime are maintained in relation to those attained by the electrodeposition in the constant potential mode.

\section{Conclusions}

Electrodeposition of copper in the potential range of the hydrogen co-deposition was examined by applying the regime of pulsating overpotential (PO) deposition in the second range. The obtained morphologies of electrodeposited copper were characterized by the scanning electron microscope (SEM). The parameters of square-wave PO were selected to enable formation of the 
honeycomb-like structures. The increase of pause duration affected neither the number nor the size of the holes formed by detachment of hydrogen bubbles. Simultaneously, the appearance of degenerate dendrites, instead of cauliflower-like agglomerates of copper grains, is observed when the pause duration was increased. The effect the PO regime in the second range on micro- and nano-structural characteristics of the honeycomb-like structures was less pronounced than that attained by the application of this regime in the millisecond range. However, the effect is more pronounced than the one observed by the electrodeposition in the constant potential mode.

Acknowledgements: The work was supported by the Ministry of Education and Science of the Republic of Serbia under the research project: "Electrochemical synthesis and characterization of nanostructured functional materials for application in new technologies" (No. 172046).

\section{References}

[1] H.-C. Shin, J. Dong, M. Liu, Adv. Mater. 15 (2003) 1610-1614.

[2] H.-C. Shin, M. Liu, Adv. Funct. Mater. 15 (2005) 582-586.

[3] J. Yin, J. Jia, L. Zhu, Int. J. Hydrogen Energy 33 (2008) 7444-7447.

[4] H.-C. Shin, M. Liu, Chem. Mater. 16 (2004) 5460-5464.

[5] N.D. Nikolić, K.I. Popov, Lj.J. Pavlović, M.G. Pavlović, J. Electroanal. Chem. 588 (2006) 8898.

[6] N.D. Nikolić, K.I. Popov, Lj.J. Pavlović, M.G. Pavlović, Surf. Coat. Technol. 201 (2006) 560566.

[7] N.D. Nikolić, K.I. Popov, Lj.J. Pavlović, M.G. Pavlović, J. Solid State Electrochem. 11 (2007) 667-675.

[8] N.D. Nikolić, Lj.J. Pavlović, M.G. Pavlović, K.I. Popov, Electrochim. Acta 52 (2007) 80968104.

[9] N.D. Nikolić, K.I. Popov, Lj.J. Pavlović, M.G. Pavlović, Sensors 7 (2007) 1-15.

[10] N.D. Nikolić, Lj.J. Pavlović, M.G. Pavlović, K.I. Popov, J. Serb. Chem. Soc. 72 (2007) 13691381.

[11] N.D. Nikolić, Lj.J. Pavlović, S.B. Krstić, M.G. Pavlović, K.I. Popov, Chem. Eng. Sci. 63 (2008) 2824-2828.

[12] N.D. Nikolić, G. Branković, M.G. Pavlović, K.I. Popov, J. Electroanal. Chem. 621 (2008) 13-21.

[13] N.D. Nikolić, K.I. Popov, "Hydrogen Co-deposition Effects on the Structure of Electrodeposited Copper" in Electrodeposition: Theory and Practice, Series: Modern Aspects of Electrochemistry, S.S. Djokić, Ed., Vol. 48, Springer (2010) pp. $1-70$.

[14] S. Li, R. Furberg, M.S. Toprak, B. Palm, M. Muhammed, Adv. Funct. Mater. 18 (2008) 22152220.

[15] R. Kim, D. Han, D. Nam, J. Kim, H. Kwon, J. Electrochem. Soc. 157 (2010) D269-D273.

[16] C.A. Marozzi, A.C. Chialvo, Electrochim. Acta 45 (2000) 2111-2120.

[17] S. Cherevko, X. Xing, C-H. Chung, Electrochem. Commun. 12 (2010) 467-470.

[18] S. Cherevko, C-H. Chung, Electrochim. Acta 55 (2010) 6383-6390.

[19] S. Cherevko, C.-H. Chung, Electrochem. Commun. 13 (2011) 16-19.

[20] S. Cherevko, X. Xing, C-H. Chung, Appl. Surf. Sci. 257 (2011) 8054-8061.

[21] Y. Li, W.-Z. Jia, Y.-Y. Song, X.H. Xia, Chem. Mater. 19 (2007) 5758-5764.

[22] J. Kim, R. Kim, H. Kwon, Electrochem. Commun. 10 (2008) 1148-1151.

[23] D. Nam, R. Kim, D. Han, J. Kim, H. Kwon, Electrochim. Acta 56 (2011) 9397-9405.

[24] N.D. Nikolić, G. Branković, M.G. Pavlović, K.I. Popov, Electrochem. Commun. 11 (2009) 421424.

[25] N.D. Nikolić, G. Branković, V.M. Maksimović, M.G. Pavlović, K.I. Popov, J. Solid State Electrochem. 14 (2010) 331-338. 
[26] N.D. Nikolić, G. Branković, V.M. Maksimović, M.G. Pavlović, K.I. Popov, J. Electroanal. Chem. 635 (2009) 111-119.

[27] N.D. Nikolić, V.Maksimović, M.G. Pavlović, K.I. Popov, J. Serb. Chem. Soc. 74 (2009) 689-696.

[28] N.D. Nikolić, G. Branković, Electrochem. Commun. 12 (2010) 740-744.

[29] N.D. Nikolić, G. Branković, K.I. Popov, Mater. Chem. Phys. 125 (2011) 587-594.

[30] N.D. Nikolić, G. Branković, V. Maksimović, J. Solid State Electrochem. 16 (2012) 321-328.

[31] N.D. Nikolić, G. Branković, V.M. Maksimović, J. Electroanal. Chem. 661 (2011) 309-316.

[32] N.D. Nikolić, G. Branković, M.G. Pavlović, Powder Technol. In press.

http://dx.doi.org/10.1016/i.powtec.2012.01.014.

(C) 2012 by the authors; licensee IAPC, Zagreb, Croatia. This article is an open-access article distributed under the terms and conditions of the Creative Commons Attribution license (http://creativecommons.org/licenses/by/3.0/) (cc) Br 